\title{
Focal Confluent Fibrosis in Cirrhotic Liver: Natural History Studied with Serial CT
}

Giuseppe Brancatelli ${ }^{1}, 2$

Richard L. Baron ${ }^{3}$

Michael P. Federle 2,4

Gianvincenzo Sparacia ${ }^{1}$

Karen Pealer ${ }^{2}$

Keywords: cirrhosis, CT, liver, liver fibrosis

D0I:10.2214/AJR.07.2782

Received June 26, 2007; accepted after revision October 24, 2008.

This article was presented at the 2006 meeting of the American Roentgen Ray Society, Vancouver, BC, Canada.

'Istituto di Radiologia, Università di Palermo, Via Villaermosa 29, 90139 Palermo, Italy. Address correspondence to G. Brancatelli (gbranca@yahoo.com).

${ }^{2}$ Department of Radiology, University of Pittsburgh Medical Center, Pittsburgh, PA.

${ }^{3}$ Department of Radiology, University of Chicago, Chicago, IL.

${ }^{4}$ Present address: Department of Radiology, Stanford University Medical Center, Stanford, CA.

AJR2009; 192:1341-1347

$0361-803 X / 09 / 1925-1341$

(c) American Roentgen Ray Society

OBJECTIVE. The objective of this study was to assess the long-term natural history of focal confluent fibrosis in cirrhotic liver with CT.

MATERIALS AND METHODS. Two radiologists retrospectively reviewed in consensus 118 liver CT examinations in 26 patients (19 men, seven women; age range, 32-68 years; mean age, 50 years) performed over approximately 6 years. Helical CT scans were obtained before and 30-35 and 65-70 seconds after injection of 125-150 mL of contrast medium at a rate of $4-5 \mathrm{~mL} / \mathrm{s}$. Proof of cirrhosis was based on liver transplantation $(n=6)$, biopsy $(n=$ $9)$, or imaging findings $(n=11)$. The number, location, and attenuation of fibrotic lesions and presence of trapped vessels were evaluated. Variation of hepatic retraction associated with the development of focal confluent fibrosis lesions was assessed using the ellipsoid volume formula and an arbitrary retraction index.

RESULTS. Each radiologist identified 41 focal confluent fibrosis lesions. All lesions were identified by both radiologists. Twelve patients (46\%) had a single lesion, $13(50 \%)$ had two lesions, and one $(4 \%)$ had three lesions. Thirty-four $(83 \%)$ of 41 lesions were located in segment IV, VII, or VIII. Thirty-two lesions (78\%) were hypoattenuating on unenhanced images, 25 lesions $(61 \%)$ were hypoattenuating on hepatic arterial phase images, and 20 lesions (49\%) were isoattenuating on portal venous phase images. Seven lesions (17\%) were or became hyperattenuating at follow-up on portal venous phase images. Trapped vessels were found in six lesions (15\%). The retraction index showed a significant increase over time $(r=$ $0.423, p \leq 0.0001)$.

CONCLUSION. The degree of capsule retraction associated with focal confluent fibrosis evolves with time and relates to the natural evolution of cirrhosis.

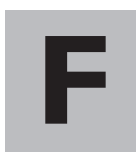

ocal confluent fibrosis is commonly encountered in patients with long-standing cirrhosis, although it has also been described in patients with early-stage compensated cirrhosis [1]. The process of hepatic parenchyma collapse and replacement with focal fibrotic masses has been shown to result in corresponding imaging changes at $\mathrm{CT}$ and MRI [2, 3]. These changes can be summarized as a focal, often wedge-shaped mass radiating from the porta hepatis, with either overlying capsule retraction or focal flattening of the capsule, most often involving the anterior and medial segment and, less frequently, the posterior segment $[2,3]$.

Investigators have reported difficulties in the MRI diagnosis of biopsy-proven hepatocellular carcinomas in cirrhotic liver because of the presence of underlying focal confluent fibrosis [4]. Other researchers have mistak- enly rendered a false-positive diagnosis of hepatocellular carcinoma at both CT [5] and MRI [6] for lesions that subsequently proved to be focal confluent fibrosis at explantation.

The organ allocation policy of the United Network of Organ Sharing based on the model for end-stage liver disease gives candidates with stage T2 hepatocellular carcinoma priority for transplantation beyond the degree of hepatic decompensation [7]. Because of the shortage of transplant organs, knowledge of the imaging characteristics of focal confluent fibrosis is important to avoid patients receiving a higher or lower priority for a transplant than is necessary. Moreover, knowledge of the presence of confluent fibrosis is important in planning liver resection because fibrosis influences the degree of liver regeneration [8].

Although the imaging findings of focal confluent fibrosis, such as capsule retraction 
and delayed enhancement, have been documented with both CT and MRI [1-3, 9], the process leading to a characteristic imaging finding has not been investigated to our knowledge. Because not all patients with confluent fibrosis show characteristic capsule retraction, we question whether those without this finding may be imaged at an earlier time and whether all patients eventually develop capsule retraction. Given the difficulties encountered by several investigators in the diagnosis of hepatocellular carcinoma when focal confluent fibrosis was present [4-6], we speculate that knowledge of the evolutionary process of focal confluent fibrosis would help correctly diagnose this entity.

The purpose of this study, therefore, was to assess the long-term natural history of focal confluent fibrosis in the cirrhotic liver with serial CT.

\section{Materials and Methods Institutional Review Board Approval and Inclusion Criteria}

This was a retrospective study performed in a single institution. Institutional review board approval was obtained for chart review. All abdominal CT reports dictated between January 1, 1998, and April 30, 2004, containing both the terms "confluent" and "fibrosis" in the text were retrospectively identified using a computerized search of our radiology information system. A research assistant and a radiologist reviewed the reports and medical records to select patients who met the inclusion criteria for this study.

The inclusion criteria were, first, a diagnosis of cirrhosis; second, CT performed after IV contrast injection; and, third, repeat liver CT performed at least once during the study period with an interval of 6 months or more between the initial and the final CT examinations. The presence of a liver tumor was not among the exclusion criteria for this study.

\section{Patients}

Twenty-six patients fulfilled the inclusion criteria: 19 men and seven women, with an age range of 32-68 years (mean age, 50 years). Women ranged in age from 40 to 59 years (mean age, 49 years), and men ranged in age from 32 to 68 years (mean age, 51 years); this difference was not statistically significant ( $p=0.547)$. All patients had cirrhosis due to the following underlying causes: alcoholism $(n=20)$; primary sclerosing cholangitis $(n=3)$; and one each of hepatitis C, HIV associated with hepatitis C, and HIV associated with hepatitis B and C. Diagnosis of cirrhosis was based on histology in 15 patients: liver transplantation $(n=6)$ and liver biopsy $(n=$ 9). In those six patients who underwent liver transplantation, the pathologists also confirmed the presence of all nine focal confluent fibrosis lesions seen at CT. In the remaining 11 patients, a clinical diagnosis of cirrhosis was based on a combination of imaging findings [10, 11], upper endoscopic findings (i.e., esophageal varices or congestive gastropathy), abnormal laboratory values (i.e., prolonged prothrombin time, decreased platelet count, and abnormal serum albumin and cholesterol levels, and increased total bilirubin and $\gamma$-globulin levels), and clinical presentation (i.e., cutaneous spider angiomas, abdominal subcutaneous portosystemic shunts, and mild ascites).

We also reviewed the Child-Pugh classification for patients, whether patients had liver neoplasms, and any related interventional procedures between the initial imaging and follow-up imaging because hepatic lesions can develop capsule retraction after treatment [12], therefore mimicking focal confluent fibrosis.

\section{CT Technique}

All CT scans were obtained at our institution with a single-detector CT scanner or a 4- or 16-MDCT scanner (HiSpeed Advantage or LightSpeed QX/I, GE Healthcare). In all patients, scanning was performed at $120-140 \mathrm{kV}$ and 200 $250 \mathrm{mAs}$ with a $512 \times 512$ matrix. For singledetector helical CT, images were obtained with a 5- to 7-mm collimation and a table pitch of 1:11:1.5. For MDCT, examinations were performed with the following parameters: collimation, 2.5 $\mathrm{mm}$; table speed, $15 \mathrm{~mm}$ per rotation; and rotation time, 0.8 second. Images were reconstructed at 5.0-mm intervals. The multiphasic CT protocol included images obtained within one breath-hold at deep inspiration during the unenhanced phase, hepatic arterial dominant phase, and portal venous dominant phase. The hepatic arterial and portal venous dominant phase scans were initiated at 30 35 and 65-70 seconds, respectively, after injection of a bolus of contrast material. All patients received 125 or $150 \mathrm{~mL}$ of either iothalamate meglumine (Conray 60, Mallinckrodt Imaging) or ioversol (Optiray 350, Mallinckrodt Imaging) injected IV at a rate of $4-5 \mathrm{~mL} / \mathrm{s}$ with a power injector (OP 100, Medrad) through an 18- or 20-gauge catheter in an antecubital vein.

\section{Image Analysis}

Two radiologists with 25 and 5 years' experience in abdominal imaging reviewed together a total of 118 examinations (range, 2-9 CT examinations per patient; range of follow-up, 3-81 months; mean CT follow-up period, 25 months; range of time interval between CT studies, 3-12 months; mean time interval between $\mathrm{CT}$ examinations, 6 months). The first CT examination during the study period ( $\sim 6$ years) was considered the initial examination, and the most recent $\mathrm{CT}$ examination was considered the final examination. The radiologists reviewed the initial CT scans as well as any follow-up scans with a PACS (Isite Radiology, Philips Healthcare) and evaluated the number, segmental location, attenuation, and size of capsule retraction associated with focal confluent fibrosis lesions. In cases of interobserver disagreement, final decisions were reached by means of consensus. All measurements were performed using settings typically close to a window width and window level of 250 and $60 \mathrm{HU}$, respectively, although in selected cases readers subjectively used a narrower window.

Focal confluent fibrosis was defined as a peripheral, wedge-shaped area showing isoattenuation or hypoattenuation compared with the adjacent liver parenchyma on unenhanced images that was associated with focal flattening or concavity of the normal convex hepatic contour. The location of focal confluent fibrosis was categorized according to the hepatic segmentation defined by the Couinaud system [13]. The overall attenuation of focal confluent fibrosis was defined relative to the liver during the same phase (unenhanced, hepatic arterial dominant, or portal venous dominant phase) of imaging. For each CT examination, two radiologists measured together with electronic calipers the greatest craniocaudal, transverse, and anteroposterior diameters of the areas of capsule retraction associated with confluent fibrosis lesions. Because coronal images were not obtained routinely during the time period of scanning, the craniocaudal diameter (height) was calculated by multiplying the thickness of the reconstructed CT images for the number of the contiguous transverse CT sections in which the focal confluent fibrosis lesion with associated retraction was detected. The transverse diameter (length) was measured as the distance between the maximum extent of the outer borders of the lesion (i.e., where the liver capsule turned from convex to flat or concave). Because focal confluent fibrosis lesions were visible on several contiguous transverse images, the image showing the largest transverse diameter was selected for measurement. The anteroposterior diameter depth (width) was calculated on the transverse image showing the largest focal confluent fibrosis transverse diameter by measuring the maximal anteroposterior distance between the deepest point of the focal confluent fibrosis lesion and an imaginary convex line joining the nonretracted liver surface adjacent to the focal confluent fibrosis lesion using an assessment of 


\section{CT of Fibrosis in the Cirrhotic Liver}

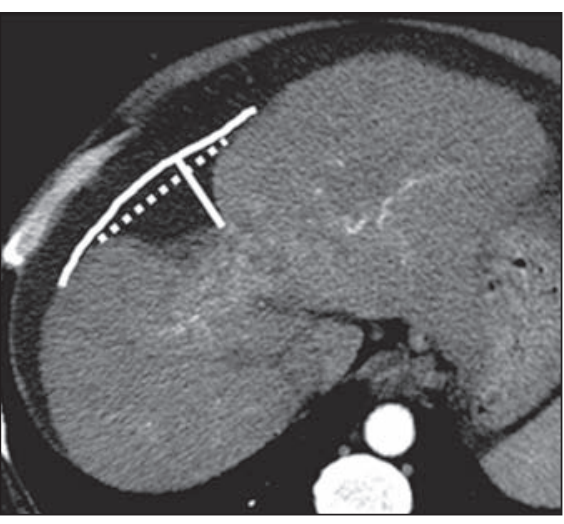

Fig. 1-CT scan shows measured parameters: dotted line $=$ transverse diameter (length), straight line = anteroposterior diameter (depth), curved line = imaginary convex line joining nonretracted liver surface adjacent to focal confluent fibrosis lesion that projects expected prior normal liver capsule course and that is used to measure anteroposterior diameter (see text).

where the normal curvature of the liver margin would have been expected to project (Fig. 1). The volume of capsule retraction associated with focal confluent fibrosis was arbitrarily calculated on the basis of the evaluated diameters using the ellipsoid volume formula: [(product of the three measurements $) \times 0.523]$. These measurements were performed on all the serial CT scans for each patient, and the results were incorporated on a standard spreadsheet.

In addition to the evolution of retraction, other secondary signs that may characterize benign fibrosis were noted: the presence of trapped vessels in the focal confluent fibrosis lesion, defined as twisted and crowded vessels seen during the arterial or portal phase, and the morphology and evolution in shape of the area of hepatic retraction toward the porta hepatis associated with the focal confluent fibrosis lesion. This area was defined as an apex when it described an angle toward the porta hepatis.

\section{Statistical Analysis}

Because multiple measurements of the ellipsoid volume were performed at irregular time intervals, a linear regression analysis with the mixed-effects model was used to correlate ellipsoid volume with time elapsed since the first $\mathrm{CT}$ examination. Statistical analysis was performed using the Fisher's exact test for slope regression $[14,15]$

Changes in retraction extent measured with an arbitrary index (retraction index) ranging from -1 to 9 , in continuous values, were obtained using standard linear regression analysis. The following equation was used to calculate the retraction index:

$124.34(a) \times$ elapsed interval in months,
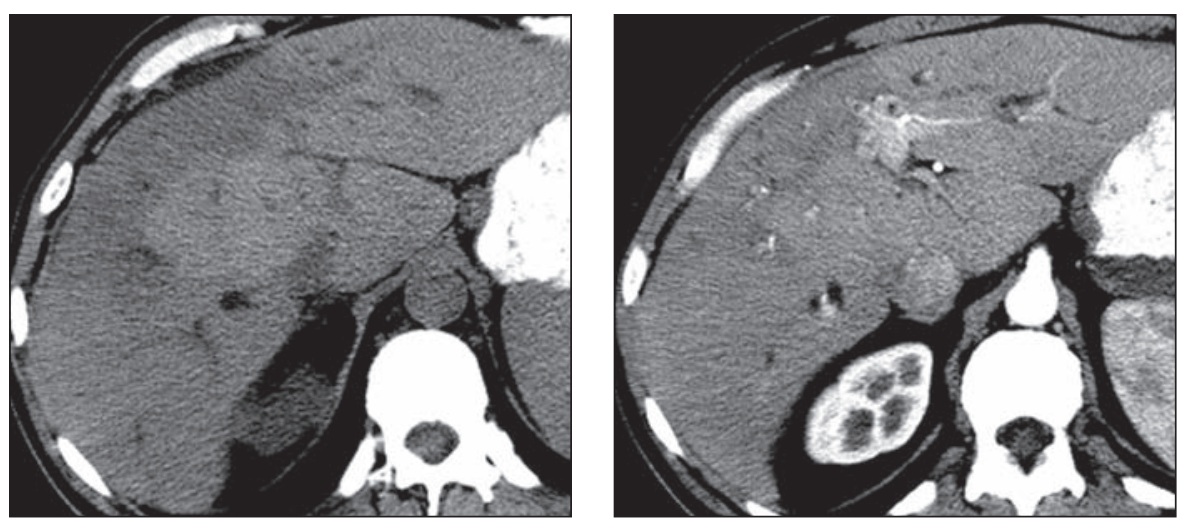

A
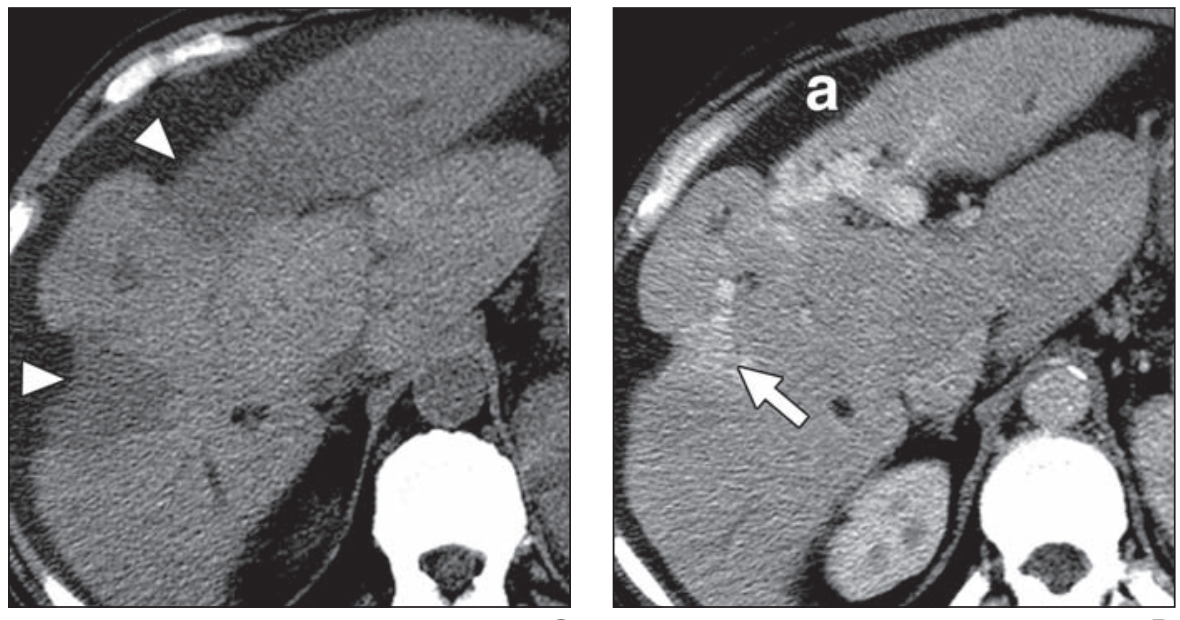

C

Fig. 2-61-year-old man with primary sclerosing cholangitis-related cirrhosis and focal confluent fibrosis. $\mathbf{A}$ and $\mathbf{B}$, Axial unenhanced (A) and contrast-enhanced (B) CT scans obtained during arterial phase show low attenuation peripherally in segments VIII and IV. No capsule retraction is seen.

$\mathbf{C}$ and $\mathbf{D}$, Unenhanced (C) and portal venous phase (D) CT scans obtained through same level as $\mathbf{A}$ and $\mathbf{B} 2$ years after $\mathbf{A}$ and $\mathbf{B}$ show focal confluent fibrosis lesions with interval development of marked atrophy and overlying capsule retraction (arrowheads, C) in segments IV and VIII. Lesion in segment VIII (arrow, D) homogeneously enhances. Retraction volume for segment VIII lesion was $13.8 \mathrm{~cm}^{3}$. Note grossly lobulated contours of liver, as typically seen in primary sclerosing cholangitis. Moderate amount of perihepatic ascites (a) is present. This patient underwent liver transplantation and diagnosis of focal confluent fibrosis was confirmed at histology.

where $a$ is the slope regression coefficient. The retraction index was defined as a variation of the ellipsoid volume over time. A stable retraction index was defined as a change in retraction of $\leq 0.5 \%$.

A classification and regression tree analysis performed using the Fisher's exact test was used to build the most efficient classification algorithm combining retraction index and time interval. Statistical analysis to compare the various clinical and demographic factors was performed using the Student's $t$ test. A $p$ value of less than 0.05 was considered significant. The unit of measure in our statistical analysis was retraction index variation, rather than the number of patients or number of lesions. All analyses were performed using Microsoft Excel 2000 and SPSS software (version 13, SPSS).

\section{Results}

Each radiologist identified 41 focal confluent fibrosis lesions. Interpretation discrepancies between the two readers were minor and were resolved by consensus. Twelve patients (46\%) had a single lesion, 13 patients $(50 \%)$ had two lesions (Fig. 2), and one patient (4\%) had three lesions. Thirty-four ( $83 \%)$ of 41 lesions were located in segment IV, VII, or VIII (Figs. 2-5). Table 1 shows the segmental locations of the lesions. Table 2 shows the attenuation of the lesions in the different phases of contrast enhancement.

Trapped vessels were found in six lesions, each in a different patient (Fig. 4). In five lesions the two radiologists noted a narrower apex in the retracted area toward the porta 


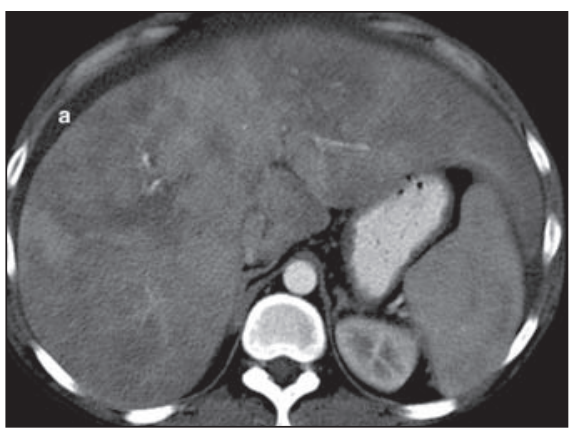

A
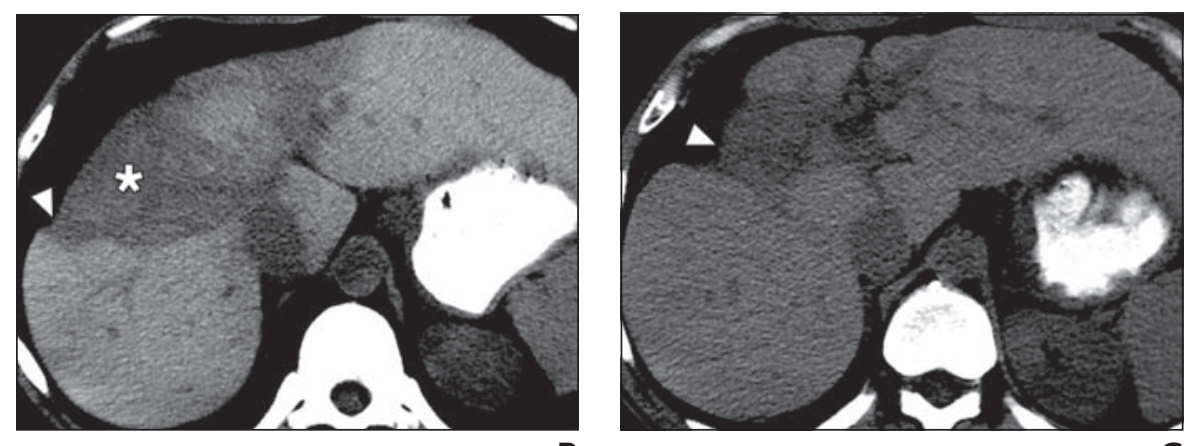

B

Fig. 3-32-year-old man with alcoholic cirrhosis and focal confluent fibrosis.

A, Axial arterial phase CT scan shows irregular, mottled liver enhancement with no evident capsule retraction. Small amount of perihepatic ascites (a) is present.

B, Axial unenhanced CT scan obtained 1 year after $\mathbf{A}$ through same level as $\mathbf{A}$ shows lesion (asterisk) of lower attenuation than adjacent liver parenchyma involving segment IV and mild retraction ( $3.3 \mathrm{~cm}^{3}$ of volume retraction) of liver capsule (arrowhead), which is typical of focal confluent fibrosis.

C, Axial unenhanced CT scan obtained 3 years after $\mathbf{A}$ shows progressive retraction of liver capsule over lesion (arrowhead) and moderate volume loss $\left(4.8 \mathrm{~cm}^{3}\right.$ of volume retraction).

hepatis, and in all cases the apex had become flattened at follow-up CT (Fig. 5).

At the initial CT (time 0), 16 patients already had some capsule retraction in 27 lesions, whereas in 10 patients the area of fibrosis was not associated with capsule retraction in 14 lesions. At follow-up CT, serial measurements showed that an increase of the retraction index was never $>52.7 \%$ (corresponding mean volume decrease $=13.8 \pm$ $7.1[\mathrm{SD}] \mathrm{cm}^{3}$ ) and that the number of lesions in which we observed an increase of retraction was never $>40 \%$ over each time interval (Table 3). The retraction index showed a progressively moderate but overall significant increase over time (Fig. 5), and capsule retraction developed in all lesions. In one patient, capsule retraction appeared to have decreased at follow-up because of the increasing shrinkage and volume loss of the adjacent liver parenchyma due to progressive lobar involvement. Table 3 shows the varia-

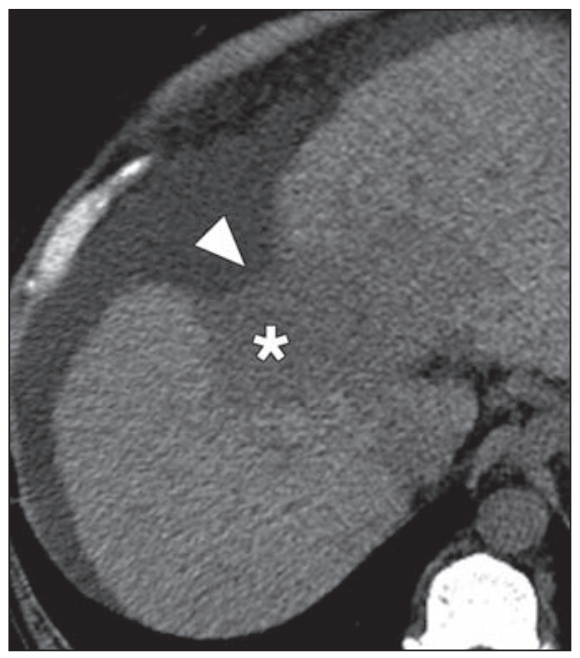

A tions of the retraction index with time. Figure 6 shows the overall significant increase of retraction index over time.

In 10 patients, cirrhosis was Child-Pugh class A, one of whom had class A/B cirrhosis. In 13 patients, cirrhosis was Child-Pugh class $\mathrm{B}$, one of whom had class $\mathrm{B} / \mathrm{C}$ cirrhosis. In three patients, cirrhosis was ChildPugh class C. Two of $26(8 \%)$ patients had a single hepatocellular carcinoma: One was treated with transarterial chemoembolization and the other with transarterial radiation therapy with ${ }^{90} \mathrm{Y}$ incorporated into glass microspheres (TheraSphere, MDS Nordion). In both cases, the hepatocellular carcinoma lesions were located in different segments of the liver from that of the focal confluent fibrosis lesions. The patient treated with transarterial chemoembolization underwent liver transplantation, and the pathologist confirmed the diagnosis of focal confluent fibrosis at explantation.

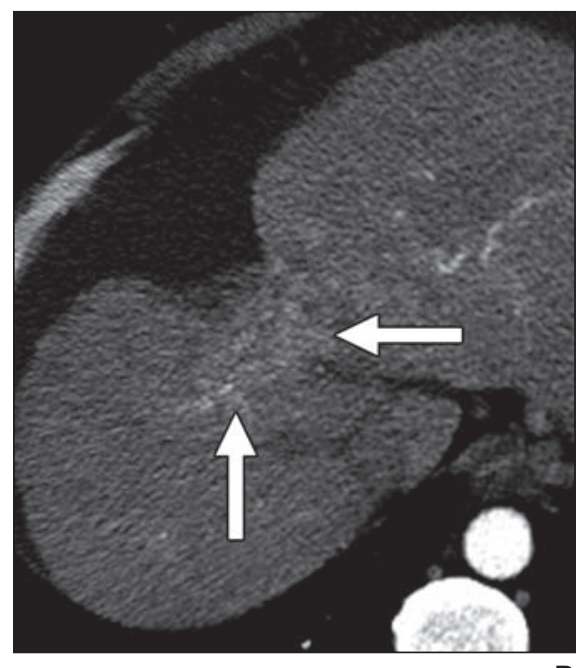

\section{Discussion}

In this study, we found that there is a progressively moderate increase over time of the capsule retraction that accompanies the development of focal confluent fibrosis. The fact that serial measurements showed an increase of the retraction index that was never $>52.7 \%$ (the corresponding mean volume decrease would have been $13.8 \pm 7.1$ [SD] $\mathrm{cm}^{3}$ ) and that the number of lesions in which we observed an increase of the retraction was never $>40 \%$ might be a consequence of the inclusion criteria of our study. Indeed, only 14 of 41 focal confluent fibrosis lesions did not show retraction at initial CT, whereas the remaining 27 already presented capsule retraction of variable severity. An explanation for this result could be that capsule retraction possibly starts developing in early-stage compensated cirrhosis [1] and that in endstage cirrhosis the architectural distortion evolves into diffuse shrinkage, which might

Fig. 4-Transverse CT scans obtained in 52-year-old man with hepatitis $\mathrm{C}$-related cirrhosis and focal confluent fibrosis.

A, Unenhanced scan shows wedge-shaped lesion (asterisk) of lower attenuation than adjacent liver parenchyma in segment VIII. Deep retraction of liver capsule (arrowhead) is seen.

B, Arterial phase scan shows trapped vessels (arrows) in focal confluent fibrosis lesions. Volume loss is $24.8 \mathrm{~cm}^{3}$. 


\section{CT of Fibrosis in the Cirrhotic Liver}
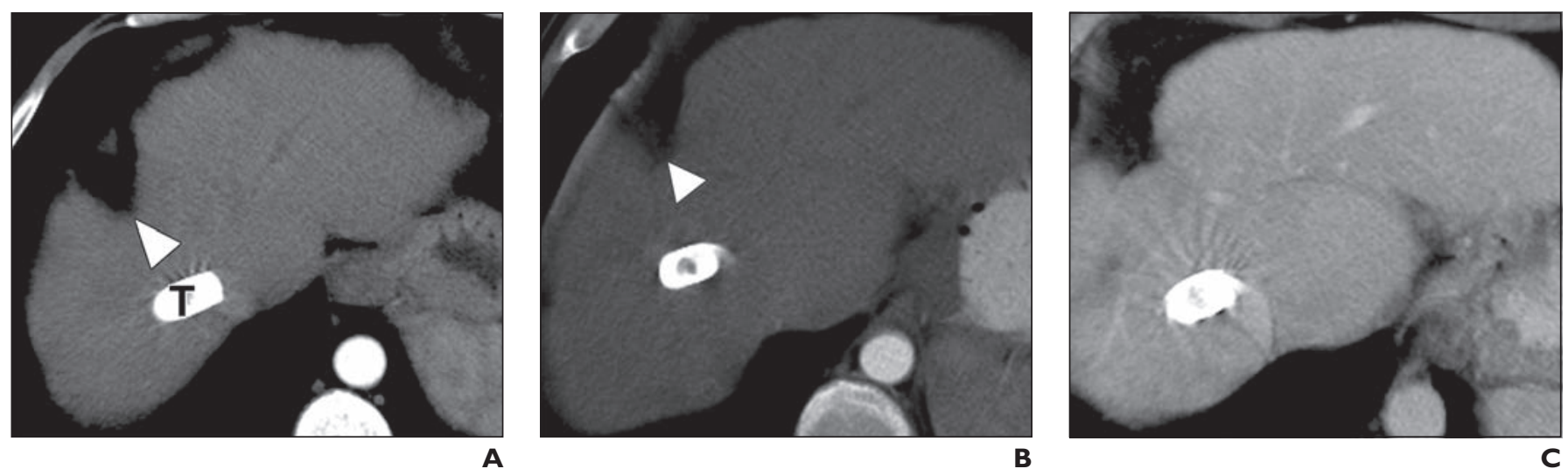

Fig. 5-48-year-old woman with alcoholic cirrhosis and focal confluent fibrosis.

A, Initial axial unenhanced CT scan shows area of retraction has shape of triangle with apex (arrowhead) oriented toward porta hepatis. Note transjugular intrahepatic portosystemic shunt (T).

B, Axial contrast-enhanced CT scan obtained 1 year after A shows apex has become slightly flattened (arrowhead). Volume loss is $2.1 \mathrm{~cm}^{3}$.

C, Axial contrast-enhanced CT scan obtained 5 years after $\mathbf{A}$ shows further flattening at apex compared with $\mathbf{A}$ and $\mathbf{B}$ and area of retraction has become polygonalshaped. Volume loss is $2.7 \mathrm{~cm}^{3}$.

TABLE I: Segmental Location of Focal Confluent Fibrosis Lesions

\begin{tabular}{|c|c|}
\hline Hepatic Segment & No. of Lesions \\
\hline VIII & 16 \\
\hline IV & 11 \\
\hline VII & 7 \\
\hline II & 5 \\
\hline VI & 2 \\
\hline Total & 41 \\
\hline
\end{tabular}

result in a reduction of capsule retraction or, as described in one of our cases, in its disappearance. Despite the relative heterogeneity of our study population and results, the retraction index showed an overall significant increase over time. We did not perform a subgroup analysis of the 14 focal confluent fibrosis lesions that did not show retraction at initial CT; therefore, we believe that a prospective study evaluating the morphology evolution of a larger number of cirrhotic livers at the initial stage - that is, when capsule retraction has not developed yet-would be beneficial in further clarifying this issue.

Ohtomo et al. [2] found that five of 49 lesions of wedge-shaped confluent fibrosis were not associated with capsule retraction. The percentages of cases with atrophy or retraction reported by those authors [2] reflect, however, only one point in time. Our study is the first, to our knowledge, to show the effects over time. In our study, even the 14 focal confluent fibrosis lesions that did not show retraction at the initial CT eventually devel-

\section{TABLE 2: Attenuation of the Focal Confluent Fibrosis Lesions in the} Different Phases of Contrast Enhancement

\begin{tabular}{l|c|c|c}
\hline \multicolumn{1}{c|}{ Phase } & Hypoattenuating & Isoattenuating & Hyperattenuating \\
\hline Unenhanced phase & 32 & 9 & 0 \\
Hepatic arterial phase $^{\mathrm{a}}$ & 25 & 15 & 0 \\
Portal venous phase $^{\prime}$ & 19 & $20^{\mathrm{b}}$ & 2 \\
\hline
\end{tabular}

aln one case, arterial phase was not obtained.

${ }^{\mathrm{b}}$ Five isoattenuating lesions became hyperattenuating at follow-up CT.

TABLE 3: Variation of Retraction Index with Time

\begin{tabular}{|c|c|c|c|c|}
\hline \multirow[b]{2}{*}{$\begin{array}{l}\text { Time of Follow-Up } \\
\text { (mo) }\end{array}$} & \multicolumn{2}{|c|}{ No. $(\%)$ of Lesions } & \multirow{2}{*}{$\begin{array}{l}\text { Average (Range) } \\
\text { of Increase in } \\
\text { Retraction Index } \\
(\%)^{b}\end{array}$} & \multirow{2}{*}{$\begin{array}{c}\text { Mean Volume } \\
( \pm \mathrm{SD}) \text { of Capsule } \\
\text { Retraction }\left(\mathrm{cm}^{3}\right)^{b}\end{array}$} \\
\hline & $\begin{array}{l}\text { Stable Retraction } \\
\text { Index }\end{array}$ & $\begin{array}{c}\text { Increased } \\
\text { Retraction Index }\end{array}$ & & \\
\hline$<24$ & $24(59)$ & $17(41)$ & $1(0.5-2.5)$ & $0.3( \pm 0.1)$ \\
\hline $24-40$ & $27(66)$ & $14(34)$ & $7(6.9-7.9)$ & $2 \quad( \pm 0.2)$ \\
\hline $40-60$ & $34(83)$ & $7(17)$ & $52(51.5-52.7)$ & $13.8( \pm 7.1)$ \\
\hline$>60$ & $36(88)$ & $5(12)$ & $17(16.8-18.9)$ & $2.2( \pm 0.4)$ \\
\hline
\end{tabular}

aRetraction index is an arbitrary index used to measure variation of hepatic retraction associated with development of focal confluent fibrosis lesion.

${ }^{b}$ In lesions that retracted.

oped retraction at follow-up CT. Our results, when considered sequentially, suggest that a higher percentage of cases will show this finding. We hypothesize that the five cases not associated with retraction described by Ohtomo et al. most likely would have eventually developed it if followed further.

In our experience, wedge-shaped areas of hypoattenuation are frequently encountered at $\mathrm{CT}$ in the surveillance of the cirrhotic liver performed every 6-12 months [16] and do not warrant biopsy when worrisome findings for hepatocellular carcinoma, such as enhancement in the arterial phase, washout in the delayed phase, and bulging of the liver capsule, are absent. Development of capsule retraction over time and the associated ancillary findings that we report here are an additional clue toward the diagnosis of focal confluent fibrosis.

In our study, we found a typical evolving morphologic pattern of the retracted area associated with focal confluent fibrosis. In addition to developing or increasing capsule retraction peripherally, the central apex of fibrosis also showed characteristic evolutionary changes. In five cases, the central apex of involvement widened so that rather than ap- 


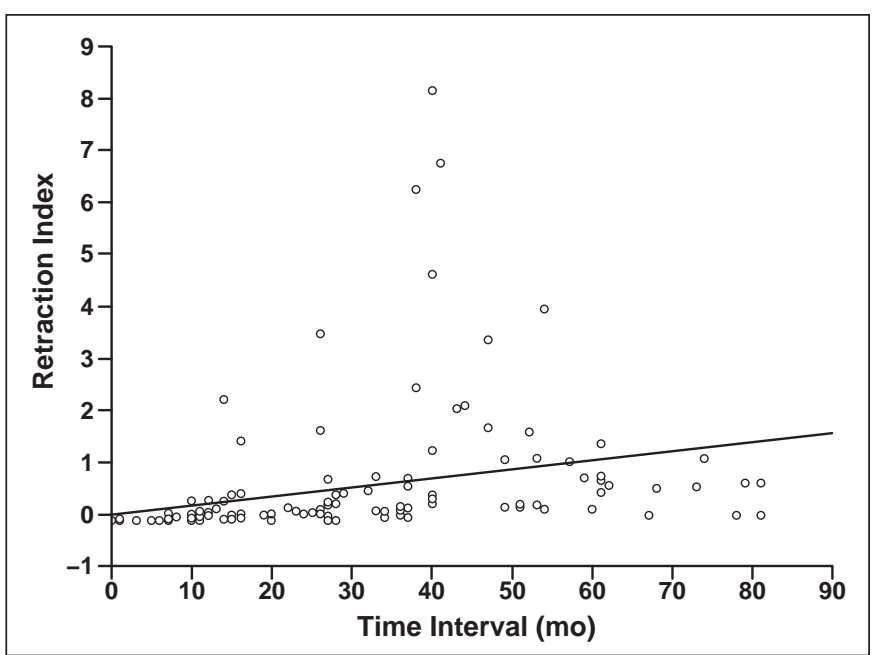

Fig. 6-Scatterplot and regression line for retraction index of 41 focal confluent fibrosis lesions measured in 26 patients. Regression line shows overall significant increase of retraction index over time.

pearing wedge-shaped, the affected area became flattened centrally. Although we did not specifically analyze the time frame of evolving changes in this subgroup of patients, we believe that an interval follow-up of 6-12 months is adequate in these patients. An awareness of these changes over time would probably further aid in differentiating these lesions from hepatocellular carcinoma.

Twenty (77\%) of our 26 patients had alcoholic cirrhosis, which is in accordance with the findings of Ohtomo et al. [2]: They found that alcohol abuse was also the type of cirrhosis most commonly associated with focal confluent fibrosis. Eighty-three percent of the lesions in our study were located in segment IV, VII, or VIII, which is also in accordance with the results of Ohtomo et al. [2, $3]$ that showed most focal fibrosis lesions involved the medial segment of the left lobe or the anterior right lobe.

Seven lesions were or became hyperdense at follow-up CT on portal venous phase imaging that were not enhancing on arterial phase imaging. This pattern of enhancement might be due to the initial retaining of contrast material by the fibrous stroma. Some authors [17, 18] have recently emphasized the importance of adding the delayed phase to the imaging protocol for the study of the cirrhotic liver, whereas others $[19,20]$ have shown that unenhanced images are of limited value. In this study we did not obtain delayed scans routinely because these articles were published after we began collecting our cases, so we do not think that we were performing a suboptimal scanning technique at that time. Nevertheless, we believe that the delayed phase is a valuable adjunct technique; therefore, in our daily practice, we have now modified our CT liver protocol accordingly. If we had performed delayed imaging in the patients in this study, we speculate that the enhancement would have been stronger [1], similar to the process of contrast retention in the fibrous stroma of cholangiocarcinoma seen on delay phase imaging [21]. Fortunately, with the exception of patients with primary sclerosing cholangitis, cholangiocarcinoma is uncommon with cirrhosis [22]. Because delayed phase enhancement and capsule retraction are imaging features commonly encountered in both cholangiocarcinoma and focal confluent fibrosis, a confident differential diagnosis with imaging alone might not be possible in patients with primary sclerosing cholangitis-related cirrhosis and therefore a biopsy should be performed.

We found trapped vessels in six lesions. Trapped vessels are likely the consequence of liver parenchymal collapse and subsequent fibrosis that does not displace vessels, but surrounds existing vessels. These vessels are often multiple and are crowded together because of the retraction and can be misdiagnosed as hepatocellular carcinoma if mistaken for tumor enhancement or tumor neovascularity. In addition, the absence of the bulging effect, usually associated with an untreated malignant lesion at the capsule surface, and absence of washout in the portal venous or delayed phase as compared with the surrounding liver are the key findings that allow differential diagnosis of hepatocellular carcinoma. The trapping of vessels within the collapsed parenchyma can be an aid to characterize fibrosis because most malignant lesions will displace vessels. Most often these crowded, trapped vessels have a different appearance than the previously described arteri- al phase enhancement that can be associated with confluent fibrosis. The small number of patients, after dividing them into subgroups, prevented us from analyzing whether a specific cause was more frequently associated with the occurrence of trapped vessels, and whether trapped vessels were observed more commonly in patients with a single lesion or multiple lesions. We are not aware of previous descriptions of trapped vessels and evolution toward an apex morphology found in association with focal confluent fibrosis.

In one of the two patients with hepatocellular carcinoma who underwent interventional treatment, the pathologist confirmed the diagnosis of focal confluent fibrosis at explantation. For the other patient with hepatocellular carcinoma, we do not have pathologic proof of the focal confluent fibrosis lesion. However, in this patient the hepatocellular carcinoma and the focal confluent fibrosis lesion were located in different segments. In previous studies in which investigators examined resected specimens after an interventional treatment, they have described extensive necrosis of the tumor and sparing of adjacent hepatic parenchyma [23]. With the same reasoning, we believe it is unlikely that the area we diagnosed as focal confluent fibrosis was retracted hepatic parenchyma secondary to the interventional treatment.

Our study has several potential limitations. First, the difficulty in obtaining accurate measurements of capsule retraction on transverse CT scans alone might lead to inaccuracies in assessing for interval change, which might be better observed on coronal reconstructions as commonly performed today. Moreover, the measurements were obtained by two readers working together, whereas a more accurate method would have been to obtain an average value of measurements calculated by three different readers. Second, the diagnosis of cirrhosis was established with pathology in only 15 patients, and the diagnosis of focal confluent fibrosis was established with pathology in only nine cases occurring in the six patients who underwent liver transplantation. In the remaining cases, biopsy of the cirrhotic liver was performed randomly and was not directed to the area of fibrosis. Third, ours was a retrospective study; therefore, some selection bias may be present. For example, the search methods we used may not have identified all patients with focal confluent fibrosis lesions who underwent scanning during the specified time period, particularly if the original interpreting radiologist did not include 


\section{CT of Fibrosis in the Cirrhotic Liver}

the term "confluent" in the official report. Fourth, the chosen 6-month interval is arbitrary. According to our referring clinicians' protocol, CT evaluations were performed every 6 months in patients with cirrhosis for hepatocellular carcinoma surveillance. Fifth, the time for follow-up examination was not standardized, and it was not possible to assess accurately the time interval between the beginning liver disease and imaging. Sixth, our CT protocol was not uniform because of the remarkable advancements in CT hardware and software in the past few years and because our study was retrospective. Seventh, we did not correlate the rate of retraction with liver function test results or other clinical parameters, and it will be of interest in future studies to investigate whether these two features are correlated. Eighth, the measurements of the depth of retraction might have been affected by the progressive shrinkage and rotation of different liver segments observed during the progression of the disease.

In conclusion, the results of our study show that the degree of capsule retraction associated with focal confluent fibrosis evolves with time and relates to the natural evolution of cirrhosis. In addition, the presence of crowded, trapped vessels within confluent fibrosis can occasionally be seen. Understanding of this process may aid in differentiating this lesion from hepatocellular carcinoma.

\section{References}

1. Kelekis NL, Makri E, Vassiou A, Patsiaoura K, Spiridakis M, Dalekos GN. Confluent hepatic fibrosis as the presenting imaging sign in nonadvanced alcoholic cirrhosis. Clin Imaging 2004; 28:124-127

2. Ohtomo K, Baron RL, Dodd GD 3rd, et al. Confluent hepatic fibrosis in advanced cirrhosis: appearance at CT. Radiology 1993; 188:31-35
3. Ohtomo K, Baron RL, Dodd GD 3rd, Federle MP, Ohtomo Y, Confer SR. Confluent hepatic fibrosis in advanced cirrhosis: evaluation with MR imaging. Radiology 1993; 189:871-874

4. Hussain HK, Syed I, Nghiem HV, et al. T2weighted MR imaging in the assessment of cirrhotic liver. Radiology 2004; 230:637-644

5. Brancatelli G, Baron RL, Peterson MS, Marsh W. Helical CT screening for hepatocellular carcinoma in patients with cirrhosis: frequency and causes of false-positive interpretation. AJR 2003 ; 180:1007-1014

6. Krinsky GA, Lee VS, Theise ND, et al. Hepatocellular carcinoma and dysplastic nodules in patients with cirrhosis: prospective diagnosis with MR imaging and explantation correlation. Radiology 2001; 219:445-454

7. Said A, Lucey MR. Liver transplantation: an update. Curr Opin Gastroenterol 2006; 22:272-278

8. Tanaka H, Hirohashi K, Kubo S, et al. Preoperative portal vein embolization improves prognosis after right hepatectomy for hepatocellular carcinoma in patients with impaired hepatic function. Br J Surg 2000; 87:879-882

9. Ooi CG, Chan KL, Peh WC, Saing H, Ngan H. Confluent hepatic fibrosis in monozygotic twins. Pediatr Radiol 1999; 29:53-55

10. Awaya H, Mitchell DG, Kamishima T, Holland G, Ito K, Matsumoto T. Cirrhosis: modified caudateright lobe ratio. Radiology 2002; 224:769-774

11. Ito K, Mitchell DG, Gabata T, Hussain SM. Expanded gallbladder fossa: simple MR imaging sign of cirrhosis. Radiology 1999; 211:723-726

12. Ernst O, Sergent G, Mizrahi D, Delemazure O, Paris JC, L'Herminé C. Treatment of hepatocellular carcinoma by transcatheter arterial chemoembolization: comparison of planned periodic chemoembolization and chemoembolization based on tumor response. AJR 1999; 172:59-64

13. Couinaud C. Le foie: études anatomiques et chirurgicales. Paris, France: Masson, 1957:9-12 14. Zhang H, Singer B. Recursive partitioning in the health sciences. New York, NY: Springer-Verlag, 1999

15. Brieman L, Friedman JH, Olshen RA, Stone CJ. Classification and regression trees. Belmont, CA: Wadsworth International Group, 1993:36-67

16. Trevisani F, De NS, Rapaccini G, et al. Semiannual and annual surveillance of cirrhotic patients for hepatocellular carcinoma: effects on cancer stage and patient survival (Italian experience). Am J Gastroenterol 2002; 97:734-744

17. Ronzoni A, Artioli D, Scardina R, et al. Role of MDCT in the diagnosis of hepatocellular carcinoma in patients with cirrhosis undergoing orthotopic liver transplantation. AJR 2007; 189:792798

18. Monzawa S, Ichikawa T, Nakajima H, Kitanaka Y, Omata K, Araki T. Dynamic CT for detecting small hepatocellular carcinoma: usefulness of delayed phase imaging. AJR 2007; 188:147-153

19. Doyle DJ, O'Malley ME, Jang HJ, Jhaveri K. Value of the unenhanced phase for detection of hepatocellular carcinomas $3 \mathrm{~cm}$ or less when performing multiphase computed tomography in patients with cirrhosis. J Comput Assist Tomogr 2007; 31:86-92

20. Iannaccone R, Laghi A, Catalano C, et al. Hepatocellular carcinoma: role of unenhanced and delayed phase multi-detector row helical CT in patients with cirrhosis. Radiology 2005; 234:460-467

21. Lacomis JM, Baron RL, Oliver JH 3rd, Nalesnik MA, Federle MP. Cholangiocarcinoma: delayed CT contrast enhancement patterns. Radiology 1997; 203:98-104

22. Welzel TM, Graubard BI, El-Serag HB, et al. Risk factors for intrahepatic and extrahepatic cholangiocarcinoma in the United States: a populationbased case-control study. Clin Gastroenterol Hepatol 2007; 5:1221-1228

23. Lopez RR Jr, Pan SH, Hoffman AL, et al. Comparison of transarterial chemoembolization in patients with unresectable, diffuse vs focal hepatocellular carcinoma. Arch Surg 2002; 137:653-657 